\title{
EFFICIENT ALGORITHMS AND ABSTRACT DATA TYPES FOR LOCAL INCONSISTENCY ISOLATION IN FIREWALL ACLS
}

\author{
S. Pozo, A. J. Varela-Vaca, R. M. Gasca, R. Ceballos \\ Department of Computer Languages and Systems. Computer Engineering College \\ University of Seville. Avda. Reina Mercedes S/N, 41012 Seville, SPAIN \\ \{sergiopozo, ajvarela, gasca, ceball\}us.es \\ http://www.lsi.us.es/ quivir
}

Keywords: isolation, inconsistency, conflict, anomaly, firewall, acl, ruleset

\begin{abstract}
Writing and managing firewall ACLs are hard, tedious, time-consuming and error-prone tasks for a wide range of reasons. During these tasks, inconsistent rules can be introduced. An inconsistent firewall ACL implies in general a design fault, and indicates that the firewall is accepting traffic that should be denied or vice versa. This can result in severe problems such as unwanted accesses to services, denial of service, overflows, etc. However, the administrator is who ultimately decides if an inconsistent rule is a fault or not. Although many algorithms to detect and manage inconsistencies in firewall ACLs have been proposed, they have different drawbacks regarding different aspects of the consistency diagnosis problem, which can prevent their use in a wide range of real-life situations. In this paper, we review these algorithms along with their drawbacks, and propose a new divide and conquer based algorithm, which uses specialized abstract data types. The proposed algorithm returns consistency results over the original ACL. Its computational complexity is better than the current best algorithm for inconsistency isolation, as experimental results will also show.
\end{abstract}

\section{INTRODUCTION}

A firewall is a network element that controls the traversal of packets across different network segments. It is a mechanism to enforce an Access Control Policy, represented as an Access Control List (ACL).

One of the most important and frequent faults during firewall ACL design and management are inconsistencies (Wool, 2004) (Pozo2, 2008). A firewall ACL with inconsistent rules implies in general design faults, and indicates that the firewall is accepting traffic that should be denied or vice versa. This can result in severe problems such as unwanted accesses to services, denial of service, overflows, etc. ACL consistency is of extreme importance in several contexts, such as highly sensitive applications (e.g. health care). Thus, algorithms and tools to automatically isolate and characterize inconsistencies must be provided in order to give firewall administrator enough information to correct them and reduce the number of faults in firewall ACLs. In this paper we are only interested in layer 3 firewall ACLs, and thus in the five typical selectors (Taylor, 2005): protocol, source and destination IPs, and source and destination ports.

Many algorithms to isolate and characterize inconsistencies in firewall ACLs have been proposed, but it is the firewall administrator who ultimately decides which rules have to be corrected. However, these algorithms have many drawbacks regarding different aspects of the consistency diagnosis problem. One of the most important ones is that they pre-process the firewall ACL using different types of non-trivial decompositions in order to use more efficient abstract data types and techniques. However, these decomposition techniques increase the number of rules in the ACL and have worst-case exponential time and space complexity. As a consequence, results of these consistency management algorithms are given over the modified ACL. Time and space complexity of inconsistency isolation algorithms is very important, since these algorithms are being used in a new range of applications in resource-constrained devices in ubiquitous networks, such as ad-hoc network node 
real-time ACL updates, real-time IDS or IPS rule updates, etc.

To the best of our knowledge, there are only two algorithms that do not decompose the ACL: the trivial one (which is worst case $O\left(f^{2}\right)$ time complexity with the number of rules in the ACL, $f$ ); an optimization over the trivial one (Pozo2, 2008), which only improves the average and best cases by an order of magnitude in best and average cases. However, the best algorithm to date (which decompose the ACL) represents an improvement over 30 times (on average) over the trivial one (Baboescu, 2003)

In this paper we propose a rule-order independent inconsistency isolation algorithm. Our approach is based on an analysis of which data type each rule selector can store, on the design of specialized abstract data types for each one, and on divide and conquer algorithm. Worst-case computational complexity of the algorithm proposed in this paper is better in all cases than Baboescu one, as is going to be shown in both theoretical complexity analysis and experimental results with real ACLs. Furthermore, ACL pre-process is not needed by our algorithm and thus results are returned over the original, unmodified ACL.

This paper is structured as follows. In section 2, we review related works comparing them to our proposal. In section 3 we briefly analyze the internals of the consistency diagnosis problem in firewall ACLs. In section 4 we explain the methodology followed to solve the problem, and propose abstract data types (ADTs) and algorithms, with their theoretical complexity analysis. In section 5, we give experimental results with real ACLs, comparing these results with other proposals. In section 6 we give some concluding remarks.

\section{RELATED WORKS}

The closest works to ours are related with consistency isolation in general network filters. In the most recent work, (Baboescu, 2003) provides algorithms to detect inconsistencies in router filters that are worst-case 30 times (an order of magnitude) faster than $O\left(f^{2}\right)$ ones for the general case of any number of selectors per rule, where $f$ is the number of rules in the ACL. Although a theoretical complexity analysis is not provided, it improves other previous isolation algorithms for $k$ filters (Eppstein, 2001) (Hari, 2000). Baboescu proposal implies ACL decomposition as a pre-process, converting selector ranges to prefixes (Srinivasan, 1998). Nevertheless, the range to prefix conversion technique could need to split a range in several prefixes and thus the final number of rules could increase over the original ACL. In (Gupta, 1999) (Taylor, 2005), Taylor and Gupta outlined that this kind of conversion could be inefficient, because transport layer specifications vary widely (for example it possible to specify open port ranges, such as "all ports greater than 1023"). Taylor also calculated that, in the worst case, a range covering $w$-bit port numbers may require $2(w-1)$ prefixes, and that a single ACL including only two port ranges could require $2(w-1)^{2}$ entries (900 entries for 16-bit port numbers, increasing the number of rules in the ACL. Thus, inconsistency isolation results are given over the modified ACL, which is bigger and different that the original one. Baboescu also calculated ACL size increase for its data set in his paper.

Other researchers have analyzed the minimal inconsistency diagnosis problem. This problem is different to the inconsistency isolation one, since isolation is the action of finding the rules that are inconsistent with other ones, and is a polynomial problem. However, inconsistency diagnosis also implies the identification of the minimal number of rules which are the cause of the isolated inconsistencies (Pozo2, 2008), and the minimal characterization of the diagnoses among an established taxonomy (Hamed, 2006). The consistency diagnosis problem consists in the resolution of these three problems (isolation, identification, characterization) plus a correction stage (if necessary).

These researchers apply ACL decompositions in some cases, and combinatorial algorithms in others, in order to optimally solve the three problems at a time. Decompositions are used in (Al-Shaer, 2004) and in (García-Alfaro, 2008), which use ACL decorrelation (Luis, 2002). As with range to prefix conversion, ACL decorrelation increase the number of rules in the ACL and have worst-case exponential time and space complexity. As a consequence, results of the consistency management algorithms are given over the modified ACL.

Ordered Binary Decision Diagrams (OBDDs) have been used in Fireman (Yuan, 2006). Fireman authors' do not decorrelate the ACL, and thus, results are given over the original one. Note that the complexity of OBDD algorithms depends on the optimal ordering of its nodes, which is a NPComplete problem (Bollig, 1996). This results in worst case exponential complexity, as with other proposals for the consistency diagnosis problem. 


\section{CONSISTENCY IN FIREWALL ACLS}

Firewall rule-matching engines match packets in a linear way, checking rules from the first one to the last. The matching process stops once a rule has been matched, or once there are no more rules in the ACL (in this case, the firewall platform executes a predefined default action). The values of selectors (or filtering fields) between different rules can overlap, and can even be rules that are completely equal to others. An example of an ACL is presented in Fig. 2. In this example, $R 4 \subset R 3$, because all selectors of R4 are at least subsets of the same selectors of R3. However, their actions are the opposite. In this case R4 is never going to be matched in this ACL, because all packets that R4 could match are also matched by a rule with higher priority, R3. In this case, the firewall administrator must be notified, since R3 may be a faulty rule (the consequence, or the error, is that there is traffic that is denied by R3 and it may be accepted). As another example take rules $\mathrm{R} 1$ and $\mathrm{R} 2, R 1 \subset R 2$. In this case traffic that is denied by $\mathrm{R} 1$ is also accepted by $\mathrm{R} 2$. This kind of relation is used by administrators to express exceptions (the most specific rule, R1) to a general rule (R2), and is not usually considered to be a fault, because there is no error in the ACL execution.

Note that in these two examples, actions are always different (in firewalls there only two possible actions: to allow or to deny a packet). If actions were equal, there is no potential erroneous behaviour in the executed ACL, and thus there is no inconsistency. However, in this case, the relation between the rules is a redundancy, which is another kind of problem that can reduce the performance and increase the memory consumption of the rulematching engine. In this paper, we are only interested in rules that be potential faults, or inconsistent rules [4] (there could be cases where a rule is inconsistent with many others). It must be clarified that inconsistencies are order-independent and mutual. We assume that $\mathrm{ACL}_{\mathrm{f}}$ do not have redundancies (redundancies can be efficiently detected and removed (Liu, 2008))

\subsection{Problem Formalization}

A layer 3 Firewall ACL is in general a list of linearly ordered (total order) condition/action rules. Each rule firewall rule is formed by an antecedent and a (binary) consequent representing the action that must be taken once a packet matches the rule.

Let PORTSRC and PORTDST be sets of natural numbers and intervals of naturals between [0..65535] representing a port number. Le IPSRC and IPDST be two sets of valid IPv4 addresses in the octet and CIDR format (o1.o2.o3.o4/CIDR). Let $P R O T O C O L$ be a set of natural numbers in [0..255] representing a protocol number. Let $I D \geq 1$ be a natural number representing the rule priority in the ACL ( 1 is the rule with more priority). These five sets plus the $I D$ represent the typical selectors of a firewall rule [3]. Let $A C T I O N=\{$ Allow, Deny $\}$ be the binary set of possible actions for a rule consequent. Let $W=P R O T O C O L \times I P S R C \times I P D S T \times P O R T S R C \times$ PORTDST be the cartesian product of the five previous sets or selectors, which represents a 5dimensional hypercube. $W$ is the space where an antecedent of a firewall rule can be defined. Layer 7 firewalls use different selectors (e.g. a selector to express the content of a packet) and thus needs a different problem analysis.

Definition 3.1. A layer 3 firewall ACL or rule set, is defined as the cartesian product $A C L_{f}=W \times A C T I O N$, where $\left|A C L_{f}\right|=f$. A rule in $A C L_{f}$ is defined as $R_{k} \in A C L_{f}, 1 \leq k \leq f, k \in I D$ where $R_{k}\left[\right.$ PROTOCOL], $\quad R_{k}\left[\right.$ IPSRC], $R_{k}$ [IPDST], $R_{k}\left[\right.$ PORTSRC], $R_{k}[$ PORTDST] represent the corresponding selectors of the rule.

\begin{tabular}{ccccccc}
\hline Priority/ID & Protocol & Source IP & Src Port & Destination IP & Dst Port & Action \\
\hline R1 & tcp & $192.168 .1 .5 / 32$ & any & $* . * * * / 0$ & 80 & deny \\
R2 & tcp & $192.168 .1 . * / 24$ & any & $* . * * * / 0$ & 80 & allow \\
R3 & tcp & $* . * . * / 0$ & any & $172.0 .1 .10 / 32$ & 80 & allow \\
R4 & tcp & $192.168 .1 . * / 24$ & any & $172.0 .1 .10 / 32$ & 80 & deny \\
R5 & tcp & $192.168 .1 .60 / 32$ & any & $* . * * * / 0$ & 21 & deny \\
R6 & tcp & $192.168 .1 . * / 24$ & any & $* . * * * / 0$ & 21 & allow \\
R7 & tcp & $192.168 .1 . * / 24$ & any & $172.0 .1 .10 / 32$ & 21 & allow \\
R8 & tcp & $* . * . * / 0$ & any & $* . * * * / 0$ & any & deny \\
R9 & udp & $192.168 .1 . * / 24$ & any & $172.0 .1 .10 / 32$ & 53 & allow \\
R10 & udp & $* . * . * / 0$ & any & $172.0 .1 .10 / 32$ & 53 & allow \\
R11 & udp & $192.168 .2 . * / 24$ & any & $172.0 .2 . * / 24$ & any & allow \\
R12 & udp & $* . * . * / 0$ & any & $* . * * * / 0$ & any & deny \\
\hline
\end{tabular}

Figure 1: Example of a Firewall ACL 
Definition 3.2. $\mathrm{ACL}_{\mathrm{f}}$ can be trivially divided in two disjoint sets, one composed of rules with Allow action $\left(A C L_{\text {allow }}\right.$, where $\left.\left|A C L_{\text {allow }}\right|=m\right)$, and the other composed of rules with Deny action $\left(A C L_{\text {deny }}\right.$, where $\left.\left|A C L_{\text {deny }}\right|=n\right)$. Thus $A C L_{\text {allow }} \cup A C L_{\text {deny }}=A C L_{f}$ and $A C L_{\text {allow }} \cap A C L_{\text {deny }}=\varnothing$

Definition 3.3. Let the antecedent of a rule of $R_{k} \in A C L_{f}$ defined as an element or subset of $W$, $a\left(R_{k}\right) \subseteq W$. Let the consequent of a rule $R_{k} \in A C L_{f}$ be defined as $c\left(R_{k}\right)=$ Allow $\vee$ Deny. The union of the antecedents of all rules in $A C L_{\text {allow }}$ is the set $A, A=\bigcup a\left(R_{i} \in A C L_{\text {allow }}\right)$. The union of the antecedents of all rules in $A C L_{\text {deny }}$ is the set $D$, $D=\bigcup_{j} a\left(R_{j} \in A C L_{\text {deny }}\right)$

Definition 3.4. Inconsistency Detection. $a\left(R_{i} \in A C L_{\text {allow }}\right) \bigcap a\left(R_{j} \in A C L_{\text {denv }}\right) \neq \varnothing$ iff $R_{i}$ and $R_{j}$ are mutually inconsistent, $I\left(R_{i}, R_{j}\right) \mid-\perp$. Since two elements in $A C L_{f}$ representing an action and the contrary over a subset of $W$ are logically inconsistent. In the same way $A C L_{f}$ is inconsistent iff $A \cap D \neq \varnothing$. Consistency is not affected by the relative priority between rules. An inconsistency is considered to be a fault if an administrator identifies the behaviour of the executed ACL as being causing undesirable effects (or having errors).

Definition 3.5. Inconsistency Isolation. It is to find out all $R_{i} \in A C L_{\text {allow }}, R_{j} \in A C L_{\text {deny }}$ such that $I\left(R_{i}, R_{j}\right) \mid-\perp$.

The objective of the algorithm proposed in this paper is to isolate (Definition 3.4) the elements in $\mathrm{ACL}_{\text {allow }}$ and $\mathrm{ACL}_{\text {deny }}$ which are mutually inconsistent (Definition 3.5). The trivial algorithm consist in checking all pairs of rules in $\mathrm{ACL}_{\mathrm{f}}$ that are consistent with Definition 3.4, which is in $O\left(f^{2}\right)$, or to check rules in the set $A$ with rules in the set $D$

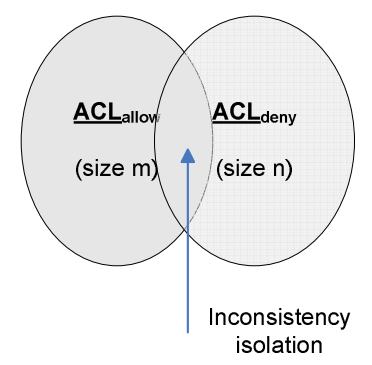

Figure 2: Result of the inconsistency isolation process

with (again with Definition 3.5), which is in $O(n \cdot m)$.

Our algorithm depart from $\mathrm{ACL}_{\text {allow }}$ and $\mathrm{ACL}_{\text {deny }}$, with $\left|A C L_{\text {deny }}\right|<\left|A C L_{\text {allow }}\right|$ (and thus $n<m)$ (if $\left|A C L_{\text {deny }}\right|<\left|A C L_{\text {allow }}\right|$, results and explanations are analogous), and is based on divide and conquer algorithm:

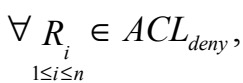

$$
\begin{aligned}
& \forall j \bullet j \in\left\{\begin{array}{l}
\text { PROTOCOL, IPSRC,IPDST, } \\
\text { PORTSRC, PORTDST }
\end{array}\right\} \bullet \\
& \sigma_{i d}\left(\prod_{j} A \cap R_{i}[j] \neq \varnothing\right)=M_{i}^{j}
\end{aligned}
$$

Each set $M_{i}$ contains the $I D$ selector of rules in $A C L_{\text {allow }}$ that intersect with a given rule $R_{i} \in A C L_{\text {deny }}$ :

$M_{i}=\bigcap_{j} M_{i}^{j}, j \in\left\{\begin{array}{l}\text { PROTOCOL, IPSRC, } \\ \text { IPDST, PORTSRC, PORTDST }\end{array}\right\}$

Thus, the result of the isolation process consists of several $M_{i}$ sets, where each one contains the IDs of the inconsistent rules in $A C L_{\text {deny }}$ for a given rule $R_{i}$ of $A C L_{\text {allow. }}$ A set $M_{i}$ is empty iff $R_{i}$ is consistent. This result can be trivially decomposed to obtain all pairs of inconsistent rules in $A C L_{f}$.

This result can be used directly by the firewall administrator, or as input to an inconsistency identification process (Pozo2, 2008), resulting in a diagnosis that can be characterized. There is a complete taxonomy for firewall ACL inconsistencies available in (Hamed, 2006). We are interested in all kinds of inconsistencies, independently of their characterization, since all of them are equally important for the firewall administrator (who is the responsible of deciding if they are considered faults or not).

\section{INCONSISTENCY ISOLATION PROCESS}

An extensive analysis of the market-leader firewall languages was presented in (Pozo1, 2009). In the analysis it was shown that IP addresses can be expressed by all of them in octets with a CIDR value (IP blocks), port numbers as naturals or intervals of naturals, and protocols as a natural number. Thus, each selector although being different by nature, can be expressed as natural numbers and, in some cases, as intervals of naturals.

One of the main ideas of our approach is to use a specialized abstract data type (ADT) to store the set of all selectors of the same type of the $m$ rules in $\mathrm{ACL}_{\text {allow }}$ (i.e. one $\mathrm{ADT}$ to store protocols used in all rules, two ADTs to store the source and destination 
IPs used in all rules, and another two ADTs to store source and destination ports). With this division and using divide and conquer, in order to know with which rules in $\mathrm{ACL}_{\text {allow }}$ the rule $R_{d} \in A C L_{d e n,}$ is inconsistent with, it is needed a search in each ADT for each selector in $R_{d}$. Each of these five searches returns all rules which have an intersecting selector. However, not all of these rules are inconsistent with $\mathrm{R}_{\mathrm{d}}$, a final combination step is needed. Attending to the presented inconsistency definitions, $R_{d}$ is inconsistent with one or more rules in $\mathrm{ACL}_{\text {allow }}$ only if all of its selectors intersect with all the selectors of

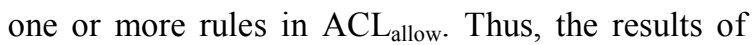
the five searches must be intersected in order to get this information. Since the process must be repeated for the $n$ rules in $\mathrm{ACL}_{\text {deny }}$, this complexity must be multiplied by $n$. The whole process is graphically represented in Fig. 3.

In the following sections, a different data structure is going to be proposed for each selector, based on the analysis of the particular data set that each one can store (Pozo1, 2009). The objective is to find or design ADTs capable of doing searches in worst case time complexity better or equal than $O(\log m)$. Finally, a combination step for these search results in worst case time complexity in $O(r)$ is also going to be proposed, where $r=m / k$, and where $k$ a very big constant $(k \geq 128)$ which is going to be theoretically calculated. So, the final time complexity of the algorithm is in worst case $O\left(f^{2} / 4 k\right)$. Note that in the worst case, $n=m=f / 2$ (i.e. $\mathrm{ACL}_{\mathrm{f}}$ has half rules with allow action, and the other half with deny). As we have said at the beginning of the section, we have assumed that $m>n$. However, if $n>m$, the algorithm can adapt itself and use $A C L_{\text {deny }}$ rules (instead of $\mathrm{ACL}_{\text {allow }}$ ones) to instantiate ADTs. That is, the algorithm always dynamically takes the bigger of the two ACLs for ADT instantiation.

Since the ADTs are only populated once, insertion time for each ADT is amortized during search operations. Also take into account that if the ACL is going to be updated (new rules are inserted, modified, or removed), ADT operations for updates are necessary. However update operations have not been considered, since updates are not the focus of this paper, but a topic for future research. The presented isolation process is thus considered static.

\subsection{ADT for protocol number selector}

Attending to the exhaustive analysis of real firewall languages presented in an earlier work (Pozo1, 2009) the protocol selector only admits 8-bit natural numbers and the wildcard, '*'. Although symbolic names are also possible, they can be converted to naturals using IANA protocol number list (RFC5237). An important fact is that no ranges are allowed in the syntax of the selector, and thus search is a trivial operation, since in order to find a non-empty intersection with a protocol number (the one of rule $R_{d} \in A C L_{\text {deny }}$ ) there are only two possible coincidences in the ADT: '*'; or exactly the same value. In the case that Rd protocol number is '*', then $\mathrm{R}_{\mathrm{d}}$ intersects with all rules of the ADT, that is all rules in $\mathrm{ACL}_{\text {allow, }}$, and no search is necessary.

To store the association <Protocol number, Rule $I D>$ we propose to use a hash table with protocol as the key, and the rule IDs as value. Hash tables

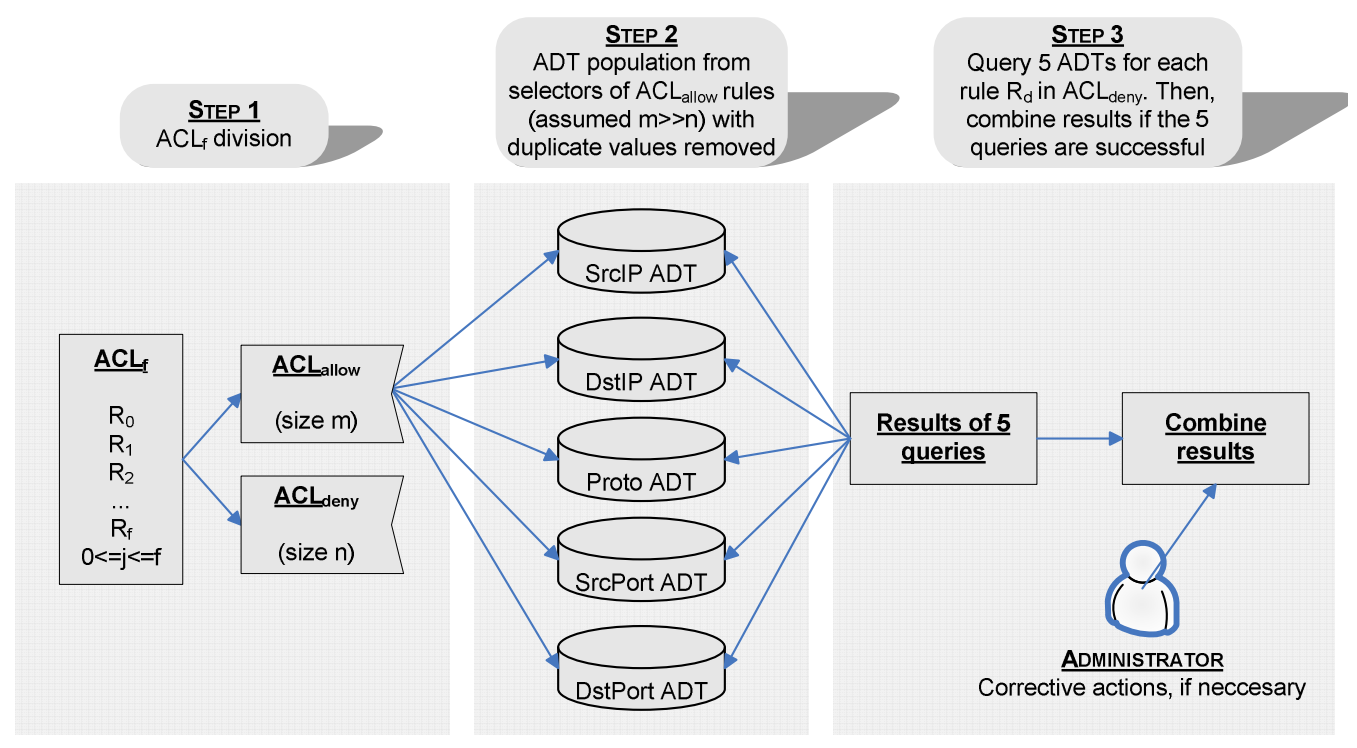

Figure 3: Proposed inconsistency isolation process 
(Cormen, 2001) have $O(1)$ (constant) time complexity for insertions, removals, updates, and search operations if a perfect hash function is used. A perfect and minimal hash function is possible, since the key space is limited and known in advance (from 0 to 65535, plus the '*'). Hash table instantiation is thus worst case $O(n)$ (the number of rules in $\mathrm{ACL}_{\text {allow}}$ ).

However, hash tables cannot store duplicate keys. This is an important problem, since in most real-life firewall ACLs only a few protocol numbers are used, although they could be thousand of rules in the ACL. This issue can be solved grouping all protocol selectors of the rules that share the same value (the same key). In this case, the associated value to the key is a set containing the rule IDs of all rules that have the key value as the value of their protocol selector. However, as removal of values could be inefficient in this way (a hash lookup plus a search in the list of rule IDs), instead of a list, it is used a fixed-size bit set of size $m$ (the size of $\mathrm{ACL}_{\text {allow }}$ ). Each position of the bit set represents one

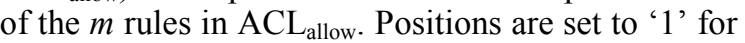
the rules in the hash table that share the same protocol number. As a side effect, with only one lookup operation in the hash table, all rule IDs that share the same protocol number are returned, as the bit set is the return result of search operations.

Fig. 4 presents the hash table associated to Fig. 1 example, and the result of all the possible search operations using the same protocol selector values of $\mathrm{ACL}_{\text {deny }}$ rules. In order to simplify the figure, only the set positions of bit sets are represented (rule IDs of the assigned rules have been directly used). Furthermore, protocol names have been transformed to IANA protocol numbers in the rightmost part of the figure.

\subsection{ADT for port number selectors}

Again, attending to the syntax analysis of market-leader firewall languages (Pozo1, 2009), the port selectors admit 16-bit natural numbers, doubleended closed natural intervals, and '*'. Symbolic names are converted to naturals or intervals. Source and destination port selectors are treated the same way from ADT and complexity viewpoint, and thus the discussion is applicable for both.

Although the only difference in syntax between protocols and ports is in the length of the natural and on the possibility of using intervals, the search operation for the port number ADT is a completely different problem.

As with the protocol selector, the result of the search operation for port numbers is all rule IDs of $\mathrm{ACL}_{\text {allow }}$ which have an intersecting port number

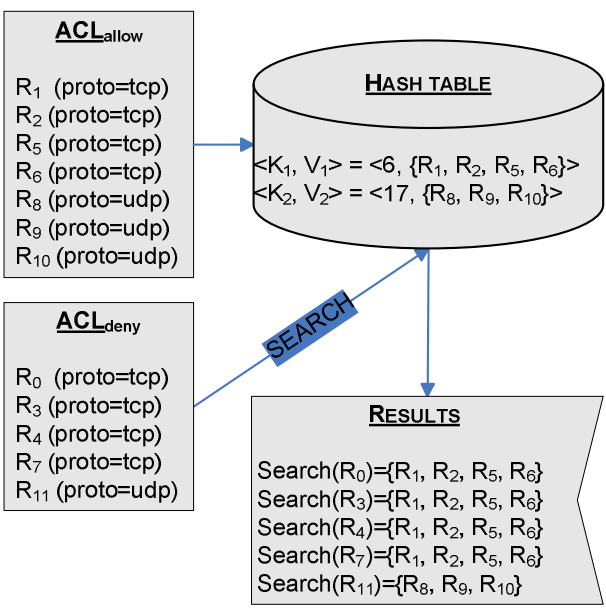

Figure 4: Hash table (perfect and minimal hash function) of protocol selector of Figure 1 example ( $\mathrm{ACL}_{\text {allow }}$ rules) and search results for $\mathrm{ACL}_{\text {deny }}$ rules

with $R_{d} \in A C L_{d e n y}$. In this case, a hash table is useless, since searching a port or an interval in it will only return equality result, but not intersections with port intervals. For example, in a hash table with keys $\{80,79-81\}$ and the port of $\mathrm{R}_{\mathrm{d}}$ is 80 , the search operation would return only the rule IDs associated to port $80 \mathrm{key}$, but note that port 80 also intersects with the interval 79-81. In the same way, if the port of $\mathrm{R}_{\mathrm{d}}$ is the interval [81-82], then no value will be returned, since the interval [81-82] is not stored in the hash table. Searching the entire hash table would return the needed result, but this operation has a linear time complexity with the number of different keys in the hash table.

There are two well-known 2D problems in computational geometry that solve similar searches (Chiang, 1991): first, given a set of data points (port numbers) and a query rectangle (port interval), give all the points that are inside the rectangle (this is the orthogonal range search problem); second, given a set of (possibly intersecting) data rectangles (port intervals) and a query point (port number), give all rectangles that intersect the query point (this is the stabbing problem).

These two 2D problems can be reformulated into 1D space, where rectangles are intervals and points are only represented by one coordinate. In $1 \mathrm{D}$, these problems are called ID range search problem (de Berg, 1997) and overlapping interval search problem (Edelsbrunner, 1983) (EdelsBrunner2, 1983) respectively. Fortunately, specialized data structures for $1 \mathrm{D}$ and 2D problems that give optimal bounds (in time and space) solutions to these two problems exist (Chiang, 1991). In the particular case of 1D, the Interval Tree (Cormen, 2001) 
(Edelsbrunner, 1983) (EdelsBrunner2, 1983), or ITree, is the selected ADT because it has optimal bound for the 1D problem (in time and space).

Fortunately, our port number or port interval search problems can trivially be reformulated to range search and overlapping interval search problems respectively, as port numbers can be represented as points in a 1D plane, and port intervals can be presented as lines in the same 1D plane.

Let $X$ be a set of $M$ points in a line, and $S$ a set of $m$ segments with endpoints in $X$. The primary structure for the ITree, $T$, can be a balanced binary search tree (Chiang, 1991) or a red-black tree (Cormen, 2001), whose internal nodes store the points of $X$, sorted from left to right, and whose leaves represent intervals between consecutive points of $X$. Each segment $s$ of $S$ is allocated at the least common ancestor of the nodes associated with the endpoints of $s$. The set of segments allocated at a node $b$, denoted by $S(b)$, is represented by two lists that store the left endpoints sorted from left to right, and the right endpoints sorted from right to left. Hence, the space complexity is in $O(m+M)$. In our problem, this is linear with the number of rules in $\mathrm{ACL}_{\text {allow. }}$ Furthermore, in our implementation duplicate intervals or points are not allowed (as with duplicate protocols), and are only stored once (again, using a bits set for rule IDs). Thus, the space complexity is reduced in a constant factor.

ITrees are static ADTs, where only a fixed set of segments and points, known in advance, can be stored. However, in order to support insertions and deletions of segments and points, the endpoint lists can be replaced with inorder-threaded balanced search trees. Hence, the update time is in amortized $O(\log m)$. Query time is in $O(\log m+L)$, where $L$ is the number of returned results (a constant factor). Thus, instantiation is in worst case amortized $O\left(m^{*} \log m\right)$, one insertion for each rule in $\mathrm{ACL}_{\text {allow. }}$. Best case time complexity could be very small when the number of port repetitions between different rules is very high, since the resulting ITree would be very small. ITrees is a well-know ADT, which has been widely used in database searches. Due to space constraints, no more information will be presented here, but it is available in the given references, including a time and space complexity analysis.

The result of the search operation over the ITree with a port number or interval of the rule $R_{d} \in A C L_{\text {deny }}$, is the union of all bit sets associated to port values in the ITree which intersect the given port of $\mathrm{R}_{\mathrm{d}}$, or a bit set with all bits set to ' 1 ' if the given port of $\mathrm{R}_{\mathrm{d}}$ is ' $*$ '. Fig. 5 presents the ITree associated to Fig. 1 example (destination port selector of $\mathrm{ACL}_{\text {allow }}$ only), and the result of all the

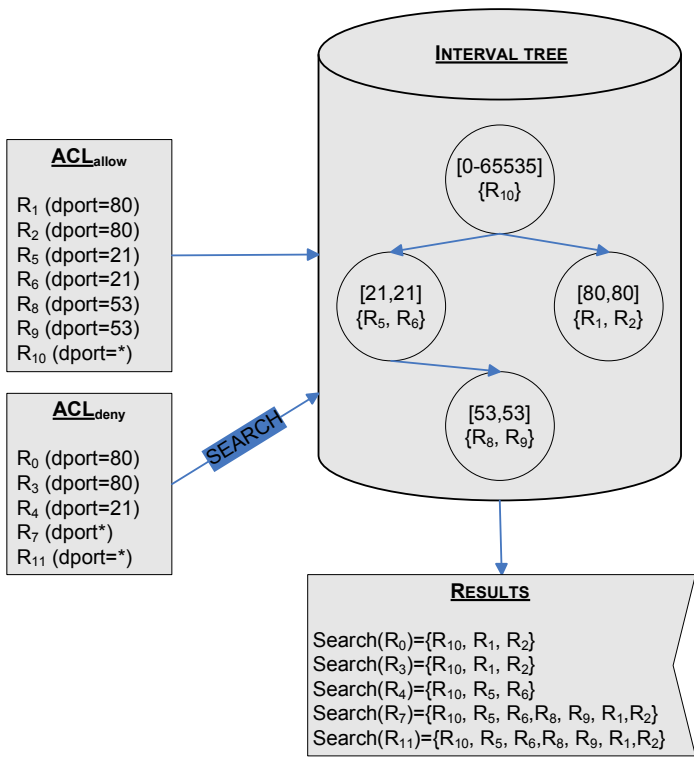

Figure 5: Interval tree of destination port selector of Figure 1 example $\left(\mathrm{ACL}_{\text {allow }}\right.$ rules) and search results for $\mathrm{ACL}_{\text {deny }}$ rules

possible search operations using destination port selector of Fig. $1 \mathrm{ACL}_{\text {deny }}$ rules.

\subsection{ADT for IP address selectors}

Attending to the syntax analysis of firewall languages (Pozo1, 2009), both IP address selectors admit 32-bit host IP addresses in CIDR format, and '*'. Symbolic names are converted to octets. Source and destination IP selectors are treated the same way from ADT and complexity viewpoint, and thus the discussion is applicable for both.

As with previous cases duplicates are not allowed (bit sets are used again). Thus, the result of the search operation must be a bit set with positions set to ' 1 ' for all rule IDs of $\mathrm{ACL}_{\text {allow }}$ which have an intersecting IP with the given in the rule $R_{d} \in A C L_{d e n v}$. As an IP block is a compact way of expressing IP address intervals, a hash table is again useless for IPs. An IP address is composed by four octets, each one being an 8-bit natural. A search operation over an ADT must use the CIDR of the IPs stored in it: Let $\mathrm{IP}_{1} / \mathrm{CIDR}_{1}$ and $\mathrm{IP}_{2} / \mathrm{CIDR}_{2}$ be two IP addresses, if $\mathrm{CIDR}_{\mathrm{S}}$ is the shortest of the two netmasks, then the intersection of $\mathrm{IP}_{1}$ and $\mathrm{IP}_{2}$ is not empty if $\mathrm{IP}_{1} \& \mathrm{CIDR}_{\mathrm{s}}=\mathrm{IP}_{2} \& \mathrm{CIDR}_{\mathrm{s}}$.

Note that valid network IP addresses have CIDR values between 1 and 30 . Value 31 is useless, since it only permits two hosts (.0 and .255 , which are not valid host IP addresses); CIDR 32 is reserved for host IPs; and CIDR 0 is only used for the wildcard IP $(0.0 .0 .0 / 0)$. 
We propose the design of a completely new and specialized ADT to store IP addresses, capable of doing searches that return multiple intersections (as with previous selectors) in time better than $O(m)$ (where $m$ is the size of $\mathrm{ACL}_{\text {allow }}$ ). As we are going to show, space complexity of this ADT is better than $O(m)$. This new ADT is called IP Tree. The general structure of an IP Tree as well as several example IPs and its corresponding IP Tree are presented in Figures 6 and 7 respectively.

The IP Tree is formed by four levels (root is not considered to be a valid level). For each node, 255 children are possible at most (0-254). These children values of each node (octets) are recursively stored in a hash table (with a perfect and minimal hash function). The association $<$ Node octet, Children nodes $>$ is called an IP Tree node, where children octets is another hash table of the same type (IP Tree node, Fig. 6).

As in the other ADTs, no repetition of IPs are allowed. Leaf nodes maintain the information regarding the IDs of the rules that share a common value for an IP address selector. In fact, leaf nodes does not have a hash table for storing $<$ Node octet, Children octets $>$ (since they do not have any children), but a hash table with a perfect hash function (there are only 30 possible CIDRs) to store $<C I D R$, RuleID Bit set $>$. CIDRs represent the CIDRs of the inserted rules that ended in that leaf, and if there are many with same CIDR (i.e. a repeated value), then bits are set to ' 1 ' in the bit set.

Insertions are done traversing the tree from top to bottom. First, the IP/CIDR address to be inserted is decomposed in its four natural octets plus the CIDR value: o1.o2.o3.o4/cidr. Then, the root node hash table is asked in order to know if $o 1$ is already in the first level of the IP Tree. If it is, the next step is to navigate to the second level through the found octet (using the children hash table). If not, a new IP Tree node with value $o 1$ is inserted in the root node children hash table. These same is done for 02,03 , and $o 4$. Once at the last level, if $o 4$ has been found, a check is launched for the CIDR data stored in the leaf $<C I D R$, Rule ID Bit set $>$ hash table using cidr value of the IP. If cidr value is found, the bit corresponding to the ID of the inserted IP is set to ' 1 '. If not, a new CIDR value is created with its corresponding bit set. Thus, the insertion of a new IP consists, in the worst case, of three $O(1)$ searches in perfect hash tables, plus a $O(1)$ search in a leaf perfect hash table, resulting in $O(1)$ worst case time complexity.

The search operation follows the same scheme as the insertion one. Note that in order to know if two IP addresses intersect, the application of the shortest netmask of the two IP addresses is necessary, as has

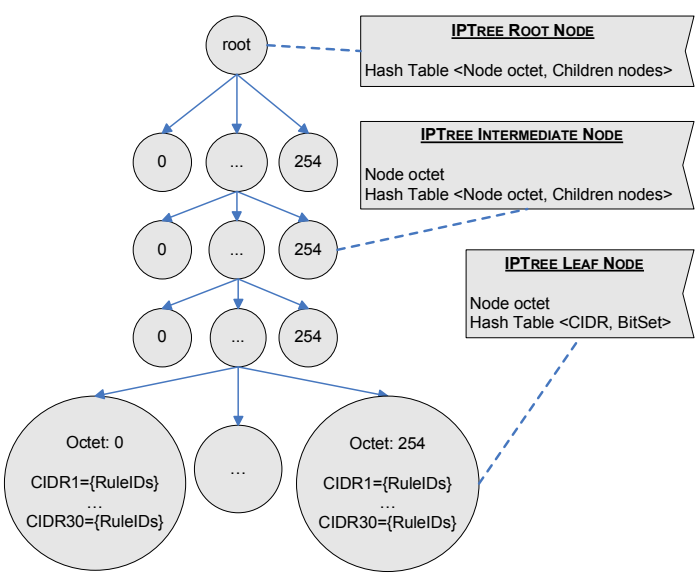

Figure 6: IPTree general structure

been pointed at the beginning of the subsection. However the result we need is the intersection of one IP with all IPs in the IP Tree, which contains all the IPs of the $m$ rules in $\mathrm{ACL}_{\text {allow. Thus, the application }}$ of all netmasks of the IPs in the IP Tree which are smaller than or equal the CIDR of the given $R_{d}$ IP address is necessary (at most 30 netmasks). The result of the application of these netmasks is a set of (at most) 30 network IPs. Now, a search operation for each of these IPs is launched. The search operation follows the same algorithm used for insertions, but taking the list of rule IDs associated to the CIDR of the leaf which coincide with the CIDR used for the search, if a search ends successfully. The result of the search operation is the union of all bit sets associated to IP addresses in the IP Tree which intersect the given IP address of $R_{d}$ (e.g. the result of the -at most- 30 searches), or a bit set with all bits set to ' 1 ' if the given IP address of $\mathrm{R}_{\mathrm{d}}$ is ' $*$ '. Note that having 30 different netmaks in a real firewall ACL is not very usual, because this usually indicates that the firewall is controlling traffic between 30 different networks, each one attached to a different physical network interface.

Thus, worst case time complexity of a search operation of a network address in a network tree is in $\mathrm{O}(30 *(4 * 1+1))=O(1)$. However, in the average case, the multiplicative factor 30 of one search operation can be reduced to $30-h$. If a search operation successfully ended in a leaf $l$, and $l$ contains $k$ CIDR values not yet used for a search in the IPTree list of CIDRs, then these values should not be used, because if used for searches, they will lead to the same leaf $l$, causing a duplicate search. This reduction of CIDRs can be made each time a new leaf is visited, thus the sum of these removed CIDRs is $h$.

Finally, host search is slightly different. Suppose that the search operation receives a host IP address 


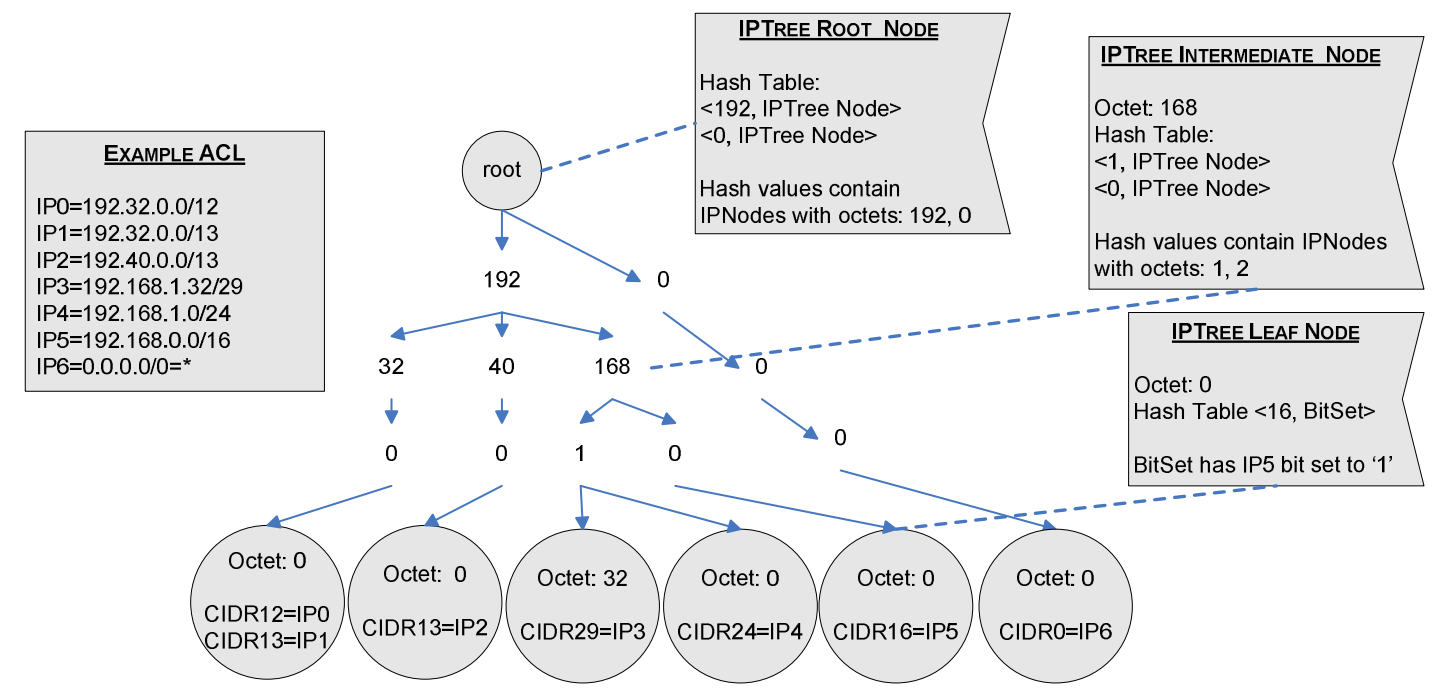

Figure 7: IPTree example for network addresses

from $R_{d}$. In this case, all CIDRs of the IP tree must be applied to the host IP, creating at most 30 IP network address. If the IP tree only contains network IP addresses, the procedure is the described above with no modifications at all. However, if the IP Tree also has host IP addresses, the new network address created from the application of a CIDR to the host IP address of $\mathrm{R}_{\mathrm{d}}$, could also intersect with another host IP address of the IP tree. This is an important problem, because the IP address of $R_{d}$ cannot intersect with more than one host IP address of the tree (the one that is exactly equal to the IP of $R_{d}$ ), although it can intersect with many network IP addresses. This multiple host IP intersection problem can be solved splitting the IP Tree in two disjoint IP trees: one to store network $\mathrm{ACL}_{\text {allow }}$ IP addresses and wildcards, and another one to store $\mathrm{ACL}_{\text {allow }}$ host IP addresses. The network IP Tree is exactly the described one (Figs. 6 and 7), but the host IP Tree is a simplified version, where no CIDR information is stored in leaves, and where all searches are exact 1..1. In addition, a slightly simplified version of insert and search methods are necessary for the host IP Tree. These simplifications are not described here due to space constraints, but could easily be derived.

\subsection{Combination of search results}

Using the calculated worst case time complexities of the search operations for the five selectors and, by the sum of the rule, the combined search time for five selectors is in worst case $O(1+2 * 1+2 * \log m)=O(\log m)$. The first factor is the time associated to the hash table search (used by protocol number selector), the second is the two searches in IP Trees (used by source and destination
IP address selectors), and the last one is the two searches in interval trees (used by source and destination port selectors).

The obtained results are five bit sets with positions set to ' 1 ' for intersecting rule IDs. However, from the inconsistency definitions, all selectors must overlap for a rule to be inconsistent with other(s). Thus, the composition of this result is somewhat trivial: the intersection of the five bit sets. This intersection gives all between $R_{d} \in A C L_{\text {deny }}$ and all rules in $\mathrm{ACL}_{\text {allow. }}$ This result can directly be used by the firewall administrator, since no decomposition has been made to the firewall ACL. Fig. 8 is an example for $R_{0} \in A C L_{\text {den, }}$, where the five bit sets resulting from the five searches for $\mathrm{R}_{0}$ selectors and their intersection are shown. Some of these results have been presented in previous figures. In this case the combination step is necessary because all searches have returned nonempty bit sets. The returned bit set indicates that $\mathrm{R}_{0}$ is inconsistent with $R_{1}$ and $R_{2}$. Now is the firewall administrator who decides if these two inconsistencies are faults or not.

As its name indicates, a bit set is an ADT whose main purpose is to store bit elements. The intersection of the five bit sets is a linear time operation with the size of the bit sets (or the number of rules in the ADTs, $m$, reduced by a constant factor derived from the duplicate removals). However, in the worst case, no repetitions are considered. Note that although the problem is linear, logical operations over bit arrays are very efficient, as they are instructions that can be executed in one machine cycle over 128 bit registers (at least) using special multi-register multimedia instructions. This yields a 


\begin{tabular}{l|c|c|c|c|c|c|c|}
\multicolumn{1}{c}{} & \multicolumn{1}{c}{$\mathbf{R}_{\mathbf{1}}$} & $\mathbf{R}_{\mathbf{2}}$ & $\mathbf{R}_{\mathbf{5}}$ & $\mathbf{R}_{\mathbf{6}}$ & $\mathbf{R}_{\mathbf{8}}$ & $\mathbf{R}_{\mathbf{9}}$ & $\mathbf{R}_{\mathbf{1 0}}$ \\
SrcIP & 1 & 1 & 1 & 1 & 1 & 1 & 0 \\
DstIP & 1 & 1 & 1 & 1 & 1 & 1 & 1 \\
SrcPort & 1 & 1 & 1 & 1 & 1 & 1 & 1 \\
DstPort & 1 & 1 & 0 & 0 & 0 & 0 & 1 \\
Protocol & 1 & 1 & 1 & 1 & 0 & 0 & 0 \\
\hline
\end{tabular}

Combination \begin{tabular}{|l|l|l|l|l|l|l|}
\hline 1 & 1 & 0 & 0 & 0 & 0 & 0 \\
\cline { 2 - 6 }
\end{tabular}

Figure 8: Combination step example

severe problem reduction by a big constant, $k \geq 128$, in time (with no space penalty).

Thus, worst case time complexity of the full process (for the $n$ rules in $\mathrm{ACL}_{\text {deny }}$ ), including the combination operation, is in worst case $O(n *(\log m+$ $m / k), \quad n=m=f / 2, \quad m / k>\log m \Rightarrow O(n * \log m)+O(n * m / k)$ $\Rightarrow O(f / 2 * \log (f / 2))+O(f / 2 *(f / 2) / k),(f / 2) / k>\log (f / 2) \quad \Rightarrow$ $O(f / 2 *(f / 2) / k) \Rightarrow O\left(\left(f^{2} / 2\right) / 2 k\right) \Rightarrow O\left(f^{2} / 4 k\right), k \geq 128$.

Derived from this analysis is the fact the complexity is bounded principally by the number of allow and deny rules (if they are equal, $n=m=f / 2$, worst case is achieved). However, as it is going to be shown in the experimental results, worst case ACLs are really unusual in the real word, where firewalls usually control traffic between small network segments with very specific services, and where multiple firewall configurations are the norm. Thus, best and average cases are achieved when there a lot of selector repetitions in $\mathrm{ACL}_{\text {allow }}$ (and thus $\mathrm{ADTs}$ are very small), when $n<<m$, and when $\mathrm{ACL}_{\mathrm{f}}$ is consistent (if a selector of a rule of $\mathrm{ACL}_{\text {deny }}$ is consistent with the same selector of all the rules of $\mathrm{ACL}_{\text {allow }}$, then that rule of $\mathrm{ACL}_{\text {deny }}$ is consistent by definition, no more searches for the rest of selectors are needed, and thus no combination of search results is needed). This results in $O(n * \log m)$, where $m$ is very small due to duplicates $\Rightarrow O(n), n<<m$.

As has also been shown, the space needed in the process is linear with the number of rules in $\mathrm{ACL}_{\text {allow }}$ plus some bit sets (the space needed to store the bit sets is negligible).
As the experimental results will show, this complexity represents (for the tested real ACLs (average cases) an algorithm that is up to three orders of magnitude faster than the trivial $O\left(f^{2}\right)$, and one to two orders faster than (Baboescu, 2003), which is the best known algorithm to date. Unfortunately, a direct theoretical comparison with Baboescu ASBV algorithm is not possible, since its time complexity is provided in number of memory accesses. The complexity reduction of our algorithm in the worst case is mainly obtained from the big multiplicative constant, $k$, and in the best case is mainly obtained from the ADTs.

\section{EXPERIMENTAL RESULTS}

In absence of standard ACLs or synthetic ACL generators, the algorithms have been tested with real firewall ACLs (Table 1).

The conducted performance analysis represents a wide spectrum of cases, with ACLs of sizes ranging from 50 to 10600 rules, and percentages of allow and deny rules ranging from $2 \%$ to $65 \%$. Recall that worst case for our proposal is achieved when half rules are allow and the other half are deny, and where all rules are inconsistent. Also note that real ACLs have some important differences with synthetically generated ones. The most important one is the number of deny and allow rules: as real firewall ACLs are usually designed with deny all default policy, most rules are going to have allow actions, and thus $\mathrm{ACL}_{\text {allow }}$ will be bigger than $A C L_{\text {deny. The result is that the worst case would not }}$ normally be achieved in real firewall ACLs.

Experiments were performed on a monothreaded Java implementation with Sun JDK 1.6.0 64-Bit Server VM, on an isolated HP Proliant 145G2 (AMD Opteron 275 2.2GHz, 2Gb RAM DDR400). Execution times are in milliseconds.

As is shown in Table 1, execution of the

Table 1: Performance evaluation

\begin{tabular}{|c|c|c|c|c|c|c|c|}
\hline $\begin{array}{l}\text { ACL } \\
\text { Size }\end{array}$ & $\begin{array}{l}\text { \%Deny } \\
\text { Rules }\end{array}$ & $\begin{array}{c}\text { No. } \\
\text { Inconsist }\end{array}$ & $\begin{array}{c}\text { Trivial } \\
\text { Isolation } \\
(\mathrm{ms}) \\
\end{array}$ & $\begin{array}{l}\text { Optimized Trivial } \\
\text { Isolation Algorithm } \\
(\mathrm{ms})\end{array}$ & $\begin{array}{c}\text { Baboescu } \\
\text { Isolation } \\
\text { Algorithm (ms) } \\
\end{array}$ & $\begin{array}{l}\text { Proposed Isolation } \\
\text { Algorithm (ms) }\end{array}$ & $\begin{array}{c}\text { ADT } \\
\text { build } \\
\text { (ms) }\end{array}$ \\
\hline 50 & 28,21 & 37 & 0.22 & 0.09 & 0.58 & 0.03 & 0.09 \\
\hline 144 & 30,91 & 108 & 1.34 & 0.62 & 1.50 & 0.06 & 0.17 \\
\hline 238 & 66,43 & 231 & 3.56 & 2.04 & 2.71 & 0.17 & 0.22 \\
\hline 450 & 34,73 & 422 & 13.22 & 5.61 & 5.29 & 0.26 & 0.54 \\
\hline 900 & 14,8 & 871 & 51.57 & 3.46 & 11.11 & 0.4 & 1.14 \\
\hline 2500 & 6,97 & 3349 & 387.86 & 55.01 & 43.12 & 0.86 & 3.54 \\
\hline 5000 & 1,98 & 4937 & 3160.09 & 64.33 & 106.99 & 1.06 & 9.02 \\
\hline 10611 & 2,05 & 11866 & 12046.67 & 332.85 & 476.81 & 8.31 & 21.85 \\
\hline
\end{tabular}


isolation process (for all rules in $\mathrm{ACL}_{\text {deny }}$ ) is really fast, even in large ACLs. If the difference between the trivial algorithm and the optimized version proposed in (Pozo2, 2008) is very big, the difference between the trivial one and the proposed in this paper is dramatic, with improvements of up to $\times 3000$ for the trivial, and up to x60 for the optimized trivial (with the test ACLs).

In the case of Baboescu ASBV algorithm, results show that his algorithm is 30 times faster than the trivial one, which also coincide with the conclusions presented in his paper. However, in practical terms, the optimized trivial algorithm presented in (Pozo2, 2008) achieves an execution time that is even a bit faster than Baboesu proposal. This is an indication of the problem increase due to the range to prefix pre-process in the Baboescu proposal.

Our proposal represents a 10 to 100 times faster alternative than the current best known one. This represents a dramatic improvement over other proposals, specially taken into account that our algorithm returns results the isolation over the original, unmodified, ACL, and not over a preprocessed one (as Baboescu proposal does).

Figure 9 presents a graphic comparison between the optimized trivial (Pozo2, 2008), Baboescu ASBV (Baboescu, 2003), and our new algorithms.

The last column in Table 1 presents ADT build time for all ADTs, showing that they are very reasonable and amortizable in a few worst-case searches. Note that once ADTs are built, they need no modification (unless the ACL changes).

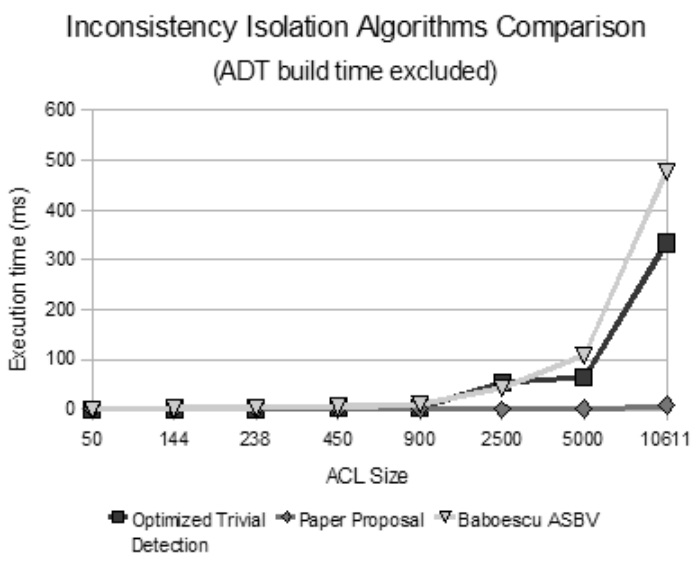

Figure 9: Execution times
The number of different values per selector and per ACL is presented in Table 2. Note that if there are a lot of values of selectors repeated in different rules of the same ACL, then search times severely improve. This is especially important for the two port selectors, since the Interval Tree is the ADT which has the worst time complexity of all ADTs. As can be seen in Table 2, the number of repetitions in the values of the selectors is unsurprisingly high in real ACLs, even if they are very big. With sufficiently small ADTs, experimental search times are near constant (this fact can be seen in Table 1, in the proposed algorithm column). The algorithms scale very well. This confirms our assumptions over real ACLs made at the end of the previous section.

\section{CONCLUSIONS}

During firewall ACL design and management inconsistencies can be introduced. An inconsistent firewall ACL implies in general a design error. However, the firewall administrator is who ultimately decides if an inconsistent rule is faulty.

In this paper, we have proposed a new inconsistency isolation algorithm for firewalls with five integer (or intervals of integer). Our approach has been based on an analysis of which data type each rule selector can to store, on the design of specialized abstract data types for each one, and on divide and conquer algorithm. A theoretical algorithmic complexity as well as an experimental performance analysis has been made in order to validate our theoretical results.

Our proposal represents an algorithm that is 10 to 100 times faster then the current best known one. Furthermore, results are returned over the original, unmodified ACL in our case, rather than over a decomposed ACL which is different than the original one.

However, our approach has some limitations that give us opportunities for improvement in future works. A performance analysis of each part ADT of the algorithm is necessary in order to know where the bottleneck is now, in order improve even more the algorithms. Checking the behaviour of the proposed ADTs in dynamic environments could be another interesting point, where another comparison in complexity and memory requirements to Baboescu algorithm would be a point. 
Table 2: Number of different elements per selector and per ACL

\begin{tabular}{|c|c|c|c|c|c|c|c|}
\hline $\mathbf{A C L}_{\mathbf{n}}$ Size & $\begin{array}{c}\text { Protocol } \\
\text { Hash Table } \\
\text { size } \\
\end{array}$ & $\begin{array}{l}\text { Source Port } \\
\text { ITree size }\end{array}$ & $\begin{array}{l}\text { Dst Port } \\
\text { ITree size }\end{array}$ & $\begin{array}{l}\text { SrcIP Host IP } \\
\text { Tree size }\end{array}$ & $\begin{array}{l}\text { SrcIP NW IP } \\
\text { Tree size }\end{array}$ & $\begin{array}{l}\text { DstIP Host IP } \\
\text { Tree size }\end{array}$ & $\begin{array}{l}\text { DstIP NW IP } \\
\text { Tree size }\end{array}$ \\
\hline 39 & 3 & 4 & 4 & 9 & 2 & 8 & 2 \\
\hline 110 & 3 & 11 & 11 & 18 & 4 & 27 & 4 \\
\hline 143 & 3 & 14 & 17 & 20 & 4 & 30 & 4 \\
\hline 334 & 3 & 19 & 26 & 29 & 4 & 38 & 4 \\
\hline 784 & 3 & 19 & 31 & 31 & 4 & 47 & 4 \\
\hline 2337 & 3 & 47 & 49 & 76 & 5 & 70 & 5 \\
\hline 4903 & 2 & 45 & 50 & 136 & 10 & 142 & 10 \\
\hline 10398 & 3 & 86 & 87 & 177 & 25 & 217 & 25 \\
\hline
\end{tabular}

Hamed, H., Al-Shaer, E. Taxonomy of Conflicts in Network Security Policies. IEEE Communications Magazine Vol.44, No.3, 2006.

\section{REFERENCES}

Al-Shaer, E., Hamed, H. Modeling and Management of Firewall Policies. IEEE eTransactions on Network and Service Management (eTNSM) Vol.1, No.1, 2004.

Baboescu, F., Varguese, G. Fast and Scalable Conflict Detection for Packet Classifiers. Computers \& Networks Vol.42, No.6, Elsevier 2003.

Bollig, B., Wegener, I. Improving the Variable Ordering of OBDDs is NP-Complete. IEEE Transactions on Computers, Vol.45 No.9, September 1996.

Cormen, T., Leiserson, C., Rivest, R., Stein, C. Introduction to Algorithms, 2nd Ed. McGraw-Hill, 2001.

Chiang, Y., Tamassia, R. Dynamic Algorithms in Computational Geometry. Technical Report CS-91-24. Brown University, Providence, RI, USA, 1991.

de Berg, M., van Kreveld, M., Overmars, M., Schwarzkopf, O. Computational Geometry: Algorithms and Applications. Springer-Verlag, Berling, 1997.

Edelsbrunner, H. A new approach to rectangle intersections, Part II. International Journal on Computational Mathematics. Vol.13, pp. 221-229, 1983.

Edelsbrunner2, H. A new approach to rectangle intersections, Part I. International Journal on Computational Mathematics. Vol.13, pp. 209-219, 1983.

Eppstein, D., Muthukrishnan, S. Internet Packet Filter Management and Rectangle Geometry. Proceedings of the Annual ACM-SIAM Symposium on Discrete Algorithms (SODA), January 2001.

García-Alfaro, J., Boulahia-Cuppens, N., Cuppens, F. Complete Analysis of Configuration Rules to Guarantee Reliable Network Security Policies, Springer-Verlag International Journal of Information Security. Vol.7, No.2, 2008.

Gupta, P., McKcown, N. Packet classification on multiple fields. Proceedings of the ACM SIGCOMM. Cambridge, MA, USA. September 1999.
Hari, B., Suri, S., Parulkar, G. Detecting and Resolving Packet Filter Conflicts. Proceedings of IEEE INFOCOM, March 2000.

Liu, Alex X., Gouda, Mohamed G., "Complete Redundancy Removal for Packet Classifiers in TCAMs," IEEE Transactions on Parallel and Distributed Systems, 24 Sept. 2008. IEEE computer Society Digital Library. IEEE Computer Society.

Luis, S., Condell, M. Security policy protocol. IETF Internet Draft IPSPSPP-01, 2002.

Pozo1, S., Ceballos, R., Gasca, R.M. Model Based Development of Firewall Rule Sets: Diagnosing Model Faults. Information and Software Technology Journal, No. 51, Issue 5, pp. 894-915. Elsevier, 2009.

Pozo2, S., Ceballos, R., Gasca, R.M.. A Heuristic Polynomial Algorithm for Local Inconsistecy Diagnosis in Firewall Rule Sets. 3rd International Conference on Security and Cryptography (SECRYPT), in International Conference on eBusiness and Telecommunications (ICETE). Porto, Portugal. INSTICC Press, 2008.

Srinivasan, V., Varguese, G, Suri, S., Waldvogel, M. Fast and Scalable Layer Four Switching. Proceedings of the ACM SIGCOMM conference on Applications, Technologies, Architectures and Protocols for Computer Communication, Vancouver, British Columbia, Canada, ACM Press, 1998.

Taylor, David E. Survey and taxonomy of packet classification techniques. ACM Computing Surveys, Vol.37, No.3, 2005.

Wool, A. A quantitative study of firewall configuration errors. IEEE Computer, Vol.37, No.6, 2004.

Yuan, L., Mai, J., Su, Z., Chen, H., Chuah,, C. Mohapatra, P. FIREMAN: A Toolkit for FIREwall Modelling and ANalysis. IEEE Symposium on Security and Privacy (S\&P’06). Oakland, CA, USA. May 2006.

\section{ACKNOWLEDGEMENTS}

This work has been partially funded by Spanish Ministry of Science and Education project under grant DPI2006-15476-C02-01, and by FEDER (under ERDF Program). 